\title{
Basins of attraction and equilibrium selection under different learning rules
}

\author{
Russell Golman • Scott E. Page
}

Published online: 28 May 2009

(C) Springer-Verlag 2009

\section{Erratum to: J Evol Econ DOI 10.1007/s00191-009-0136-x}

As stated in the article, Theorems 2 and 4 are incorrect. They refer to strict equilibrium actions in the limit $\vec{P} \rightarrow \overrightarrow{\hat{P}}$ when in fact the results only hold for pure, evolutionarily stable strategies (ESS) that are uniform in this limit. The corrected versions along with the definition of a uniformly ESS are included below.

Definition An equilibrium s is a uniformly ESS in the limit as $\vec{P} \rightarrow \overrightarrow{\hat{P}}$ if there is a punctured neighborhood $\dot{U}(\mathbf{s})$ of s such that for all $\mathbf{s}^{\prime} \in \dot{U}(\mathbf{s})$ and all $\vec{P} \neq \overrightarrow{\hat{P}}$ in some neighborhood of $\overrightarrow{\hat{P}}$,

$$
\mathbf{s} \cdot \vec{\pi}\left(\mathbf{s}^{\prime}\right)>\mathbf{s}^{\prime} \cdot \vec{\pi}\left(\mathbf{s}^{\prime}\right),
$$

where the payoff vector $\vec{\pi}=\left(\pi_{1}, \ldots, \pi_{n}\right)$.

The online version of the original article can be found at http://dx.doi.org/10.1007/s00191-009-0136-x.

R. Golman ( $\nabla)$

Department of Applied and Interdisciplinary Mathematics, University of Michigan, 2082 East Hall, 530 Church Street, Ann Arbor, MI 48109, USA

e-mail:rgolman@umich.edu

S. E. Page

Center for the Study of Complex Systems, University of Michigan, 321A West Hall, 1085 S. University Ave., Ann Arbor, MI 48109, USA

e-mail: spage@umich.edu 


\section{Theorem 2 Suppose}

$$
\lim _{\vec{P} \rightarrow \overrightarrow{\hat{P}}} \sum_{a} m(B(\mathbf{R}, a, \vec{P}) \cap B(\mathbf{B}, a, \vec{P}))=0 .
$$

Then every pure, uniformly ESS satisfies the Never an Initial Best Response Property at $\overrightarrow{\hat{P}}^{1}$

Proof Suppose that $\mathbf{s}$ is a pure, uniformly ESS such that $m\left(\mathrm{BR}^{-1}(s)\right)$ remains strictly positive in the limit $\vec{P} \rightarrow \overrightarrow{\hat{P}}$. We will identify a nonvanishing region inside the basins of attraction of $\mathbf{s}$ for both replicator dynamics and best response dynamics.

As a pure strategy equilibrium, $\mathbf{s}$ can be written as $\left(x_{s}=1, x_{-s}=0\right)$ where $s$ is the action always taken in this equilibrium. Let $U(\mathbf{s})$ be a neighborhood of s such that $\dot{U}(\mathbf{s})=U(\mathbf{s}) \backslash\{\mathbf{s}\}$ satisfies the condition for $\mathbf{s}$ to be a uniformly ESS. Let $v=\sup _{\mathbf{x} \notin U(\mathbf{s})} x_{s}$. Define the neighborhood $W(\mathbf{s}) \subseteq U(\mathbf{s})$ of all points satisfying $x_{s}>v$. We have constructed $W(\mathbf{s})$ such that $\mathbf{x} \in \dot{W}(\mathbf{s})$ implies that $\dot{x}_{s}>0$ under the replicator dynamics (because by the ESS condition, action $s$ has better than average payoff here) and in turn, $\dot{x}_{s}>0$ implies that $\mathbf{x}$ remains in $W(\mathbf{s})$.

We now observe that $\mathrm{BR}^{-1}(s)$ is a convex set because of the linearity of payoffs. Additionally, since $\mathbf{s}$ is a pure Nash Equilibrium, $\mathbf{s} \in \mathrm{BR}^{-1}(s)$. Thus, $\mathrm{BR}^{-1}(s)$ and $W(\mathbf{s})$ have positive intersection. By the fact that $W(\mathbf{s})$ is independent of $\vec{P}$ and our hypothesis that $\mathrm{BR}^{-1}(s)$ is nonvanishing, we conclude that $m\left(W(\mathbf{s}) \cap \mathrm{BR}^{-1}(s)\right)$ remains strictly positive in the limit $\vec{P} \rightarrow \overrightarrow{\hat{P}}$. Note that by the ESS condition and the linearity of payoffs, we can rule out the possibility that there are multiple best responses anywhere in the interior of $\mathrm{BR}^{-1}(s)$. For points $\mathbf{x}$ in the interior of $W(\mathbf{s}) \cap \mathrm{BR}^{-1}(s)$, best response dynamics flows to $\mathbf{s}$ because $\mathrm{BR}(\mathbf{x})=\{\mathbf{s}\}$ and replicator dynamics flows to $\mathbf{s}$ because $\mathbf{x} \in W(\mathbf{s})$.

\section{Theorem 4 Suppose}

$$
\lim _{\vec{P} \rightarrow \vec{P}} \sum_{a} m(B(\mathbf{O S P P}, a, \vec{P}) \cap B(\mathbf{T D}, a, \vec{P}))=0 .
$$

Then every pure, uniformly ESS satisfies the Never an Initial Best Response Property at $\overrightarrow{\hat{P}}$.

Proof The proof here mirrors the one for Theorem 2. We construct the neighborhood $W(\mathbf{s})$ in the same way, but with the additional condition that

\footnotetext{
${ }^{1}$ We would like to thank Andreas Blass for pointing out that this theorem, and Theorem 4 as well, would also hold for a particular action and not just for the entire action set, in the sense that if there is vanishing overlap in the basins of attraction of a particular pure equilibrium $\mathbf{s}$, then either $\mathbf{s}$ is not a uniformly ESS or $\mathbf{s}$ is almost never an initial best response.
} 
$x_{s}>1-\hat{K}$. We need only show that for $\mathbf{x} \in \operatorname{int}\left(W(\mathbf{s}) \cap \mathrm{BR}^{-1}(s)\right)$, both classes of dynamics flow to $\mathbf{s}$. Under one-sided payoff positive dynamics, $\dot{x}_{s}>0$ for $\mathbf{x} \in W(\mathbf{s})$ because action $s$ has an above average payoff, and such a flow cannot leave $W(\mathbf{s})$. Under threshold dynamics, when $\mathbf{x} \in \operatorname{int}\left(W(\mathbf{s}) \cap \mathrm{BR}^{-1}(s)\right)$, Eq. 5 applies to all actions other than $s$ because they have payoffs below the $\hat{K}^{\text {th }}$ percentile. All other actions must have the same negative growth rate, so $\dot{\mathbf{x}}=\alpha(\mathbf{s}-\mathbf{x})$ for some positive constant $\alpha$. 